Iranian Journal of Breast Diseases. 2020;13(2): 19-27.

\section{Original Article \\ Analyzing the Survival of Patients with Breast Cancer with a Multistate Model}

\author{
Najafi $\mathbf{R}^{1}$, Olfatifar $\mathbf{M}^{2}$, Razi $\mathbf{M}^{3}$, Amiri $\mathbf{F}^{4^{*}}$ \\ ${ }^{1}$ Student Research Center, Department of Biostatistics, Faculty of Health, Hamedan \\ University of Medical Sciences, Hamedan, Iran \\ ${ }^{2}$ Gastroenterology and Liver Diseases Research Center, Research Institute for \\ Gastroenterology and Liver Diseases, Shahid Beheshti University of Medical \\ Sciences, Tehran, Iran \\ ${ }^{3}$ Student Research Center, Department of Nursing, Nursing and Midwifery Faculty, \\ Hamedan University of Medical Sciences, Hamedan, Iran \\ ${ }^{4}$ Department of Biostatistics, Faculty of Medical, Arak University of Medical \\ Sciences, Arak, Iran
}

\author{
Receive: $8 / 4 / 2020$ \\ Accepted: 22/6/2020 \\ "Corresponding Author: \\ Fatemehamirii134@gmail.com \\ Ethics Approval: \\ IR.UMSHA.REC.1396.117
}

\begin{abstract}
Introduction: Breast cancer is the second most common cancer and one of the most common causes of death in women. Metastasis is also a challenge for breast cancer patients, affecting their survival. This study aimed to determine the survival rate and measure the factors affecting the progression of the disease from the time of diagnosis by considering metastasis as an intermediate condition using the disease-mortality model.
\end{abstract}

Methods: This retrospective study included 493 women with breast cancer referred to Imam Khomeini Clinic, Hamadan, between 2001 and 2017. First, the required information was extracted from patients' files according to a checklist, and then the effect of background and clinical variables on the natural course of the disease was examined using a multistate model.

Results: The risk of metastasis for people whose tumor size was $2-5 \mathrm{~cm}$ and $>5 \mathrm{~cm}$ was higher than that for people whose tumor size was $<2 \mathrm{~cm}$ (HR, 1.28; [95\% CI, 1.10-1.79]; and HR, 2.48; [95\% CI, 1.25-3.91], respectively). People with estrogen-, progesterone-, and epidermal growth factor-positive tumors were at higher risk for metastasis (HR, 2.58; [95\% CI, 1.96-3.40]; HR, 1.69; [95\% CI, 1.7-2.26]; and HR, 1.73; [95\% CI, 1.29-2.31], respectively).

Conclusion: Public education on the importance of periodic examinations and early detection of breast cancer, as well as recognizing the symptoms of the disease, can help a person detect the disease early. Therefore, the patient can see a doctor before the tumor gets bigger and the disease progresses, which will help in timely treatment of the disease.

Keywords: Breast Cancer, Multistate Model, Survival 
'مركز يزوهش دانشجويان، كروه آمار زيستى، دانشكده بهداشت، دانشخاه علوم بزشكى همدان، همدان، ايران

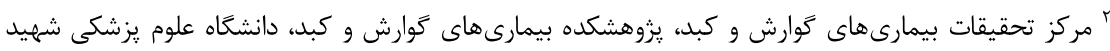
بهشتى، تهران، ايران

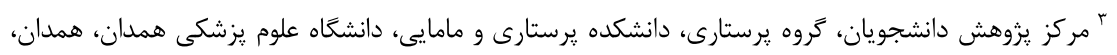

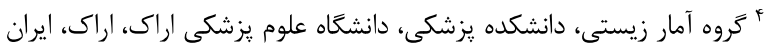

OUt?

مقدمه: سرطان يستان دومين سرطان شايع و يكى از شايعترين علل مرى در زنان است. متاستاز

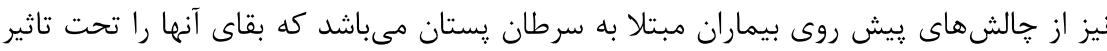

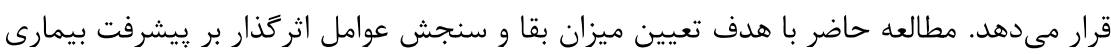

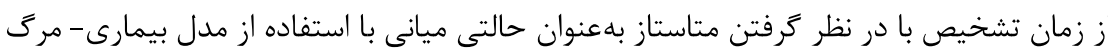
انجام شد.

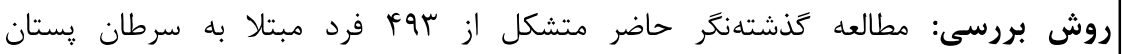

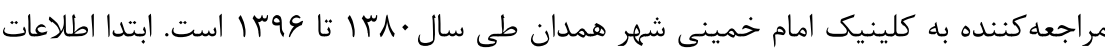

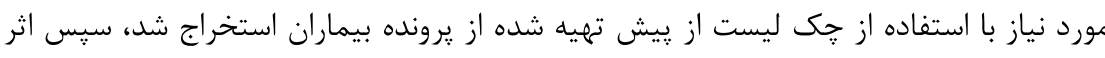

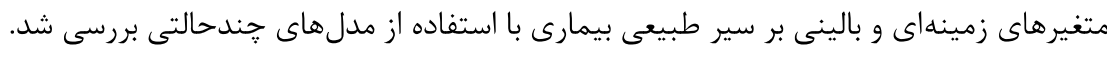

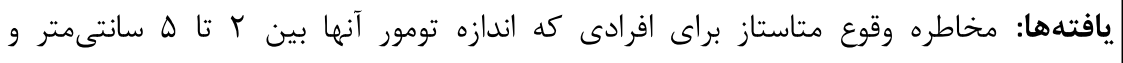

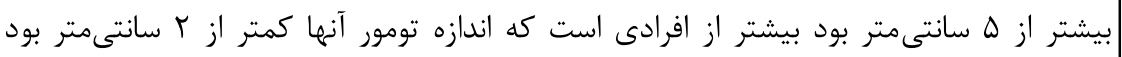

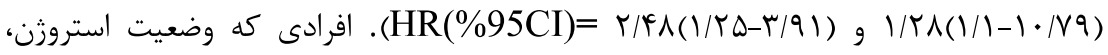

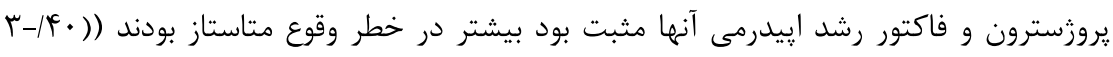

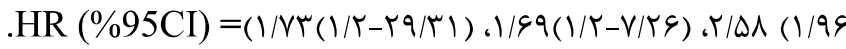
نتيجه كيرى: آموزش همعانى در مورد اهميت معاينات دورهاى و تشخيص زودهنعام سرطان

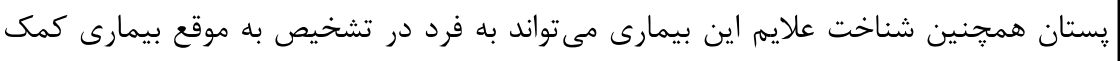

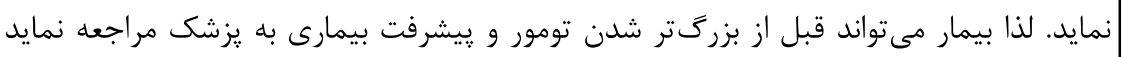

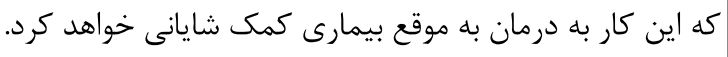
|وازههاى كليدى: سرطان پستان، مدل جند حالتى، بقا
تاريخ ارسال: • • تاريخ بريرش

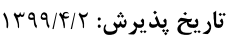

" "نويسنده مسئول: Fatemehamirii134@gmail.com 
براى هر بيمار به ترتيب شامل متغيرهاى سن در زمان

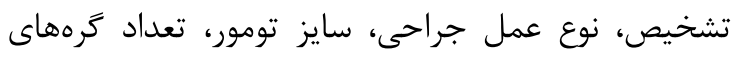

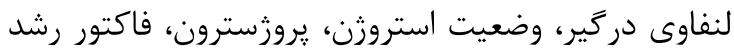

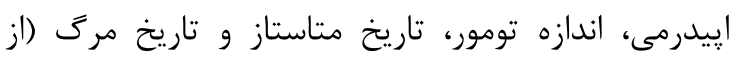

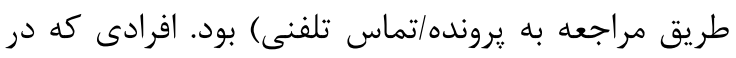
دوره بِيخيرى به هر دليلى (مهاجرت، ادامه روند درمان در برد

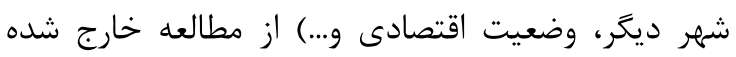

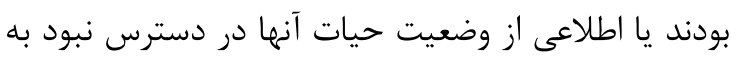
عنوان موارد سانسور شده در نظر كرفته شدند.

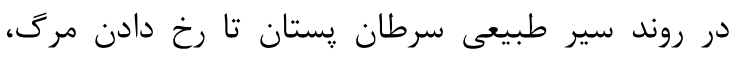

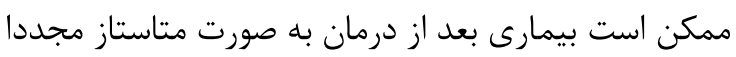

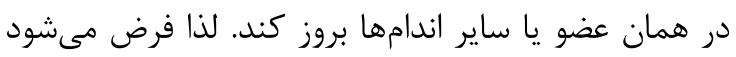

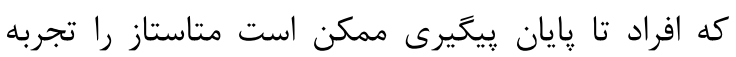

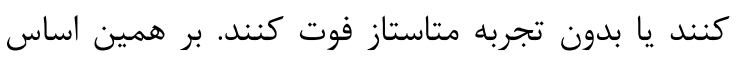

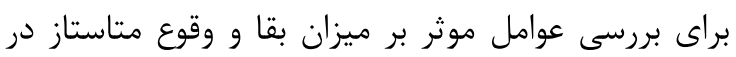

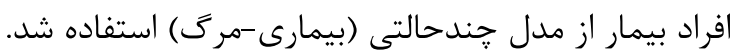

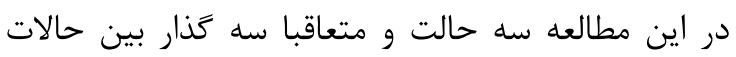

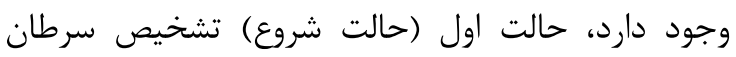

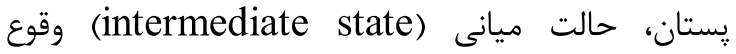
متاستاز و حالت پِايانى (حالت جذاب، مالت (absorbing state)

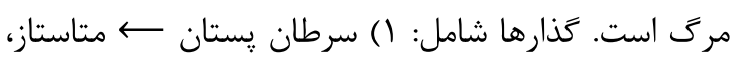

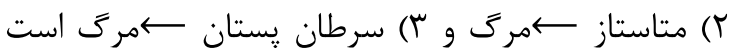

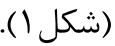

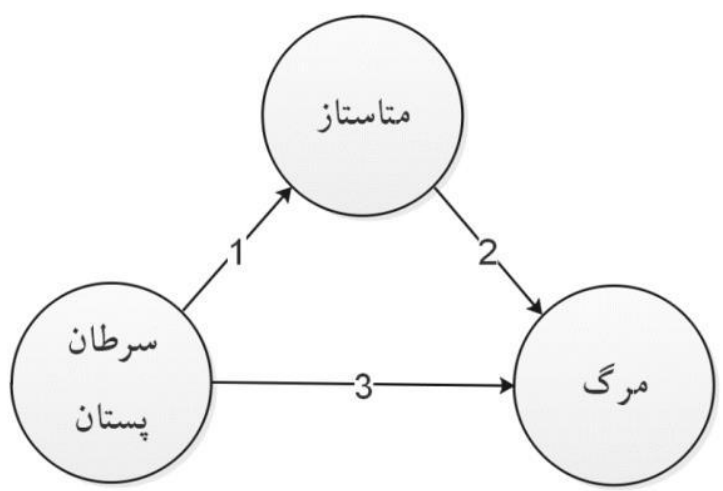

شكل ا: روند سير طبيعى(natural history) سرطان يستان

مرك در اينجا حالت جاذب است در واقع بعد از حالت

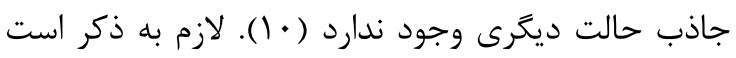
كه با توجه به طبيعت بيمارى پِّتان احتمالات كذار

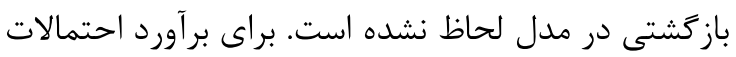

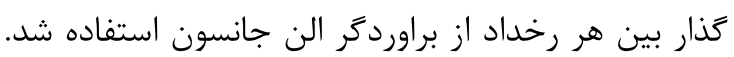

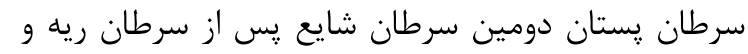

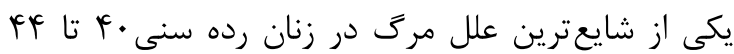

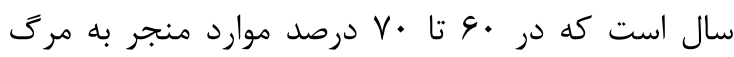
مىشود (1، Y). بيماران مبتلا به سرطان يستان كه اولين

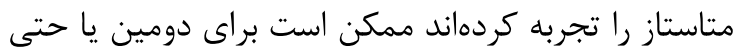

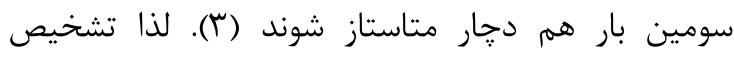

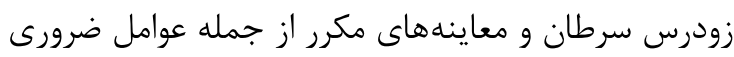
در كنترل و يِيشخيرى از سرطان يستان است (بآ).

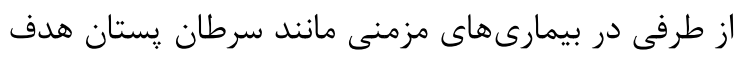

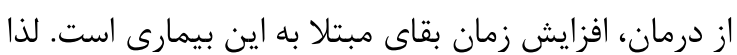
بررسى عوامل اثركذار بر زمان بقاى بيماران بسيار حايز

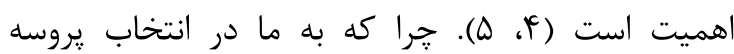
درمانى مناسب و نيز مديريت بهتر بيمارى در افراد بيمار

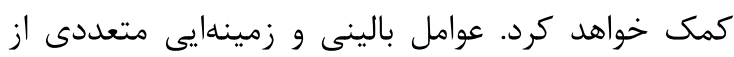
جمله سن، گسترش بيمارى به غدد لنفاوى، مرحله

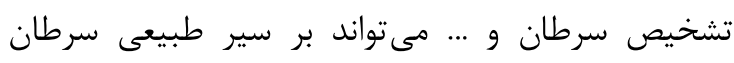
يستان و بقاى آن اثر بحذارند (و، V). لذا مطالعه و تعيين

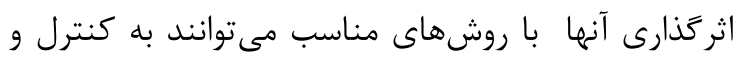
ييشخيرى سرطان يستان كمك كنند.

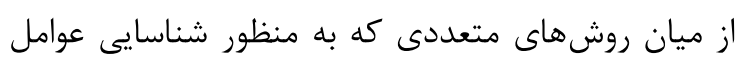

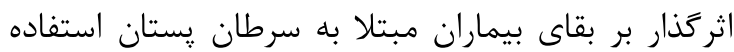

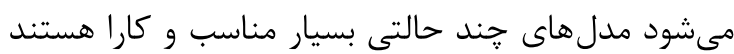

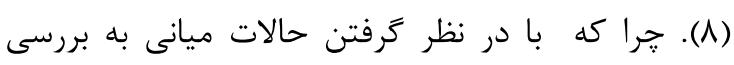

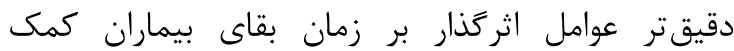
مى كنند (9). ناديده گرفتن رخداد ميانى محاسبه احتمال

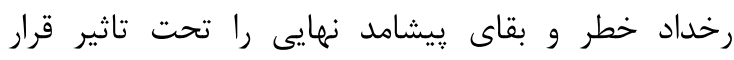

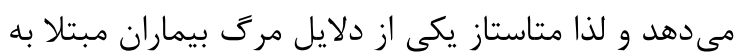
سرطان بستان است. مطالعه حاضر با هدف تعيين ميزان

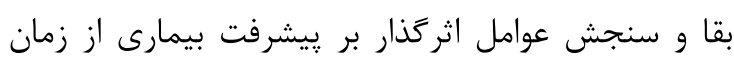

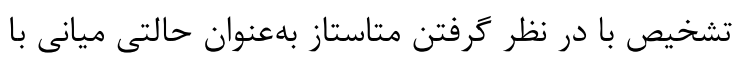
استفاده از مدل بيمارى - مرى انجام شد.

\section{مواد و روشها}

مطالعه كذشتهنكر حاضر متشكل از بو بو فرد مبتلا به

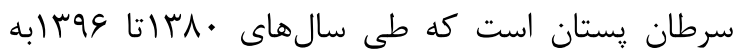
كلينيك امام خمينى در شهر همدان مراجعه كرده بودنند.

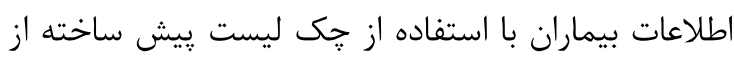
يروندهاى بالينى آنها استخراج شد. اطلاعات استخراج شده 


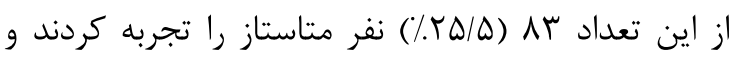

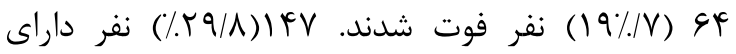

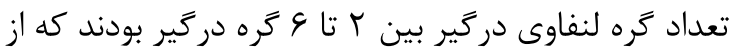

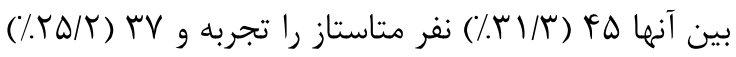
نفر نيز فوت كردند (جدول (1). در سى و ششمين ماه بِيخيرى (سه سال اول مطالعه)

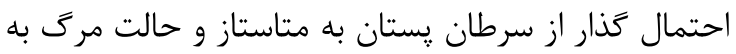

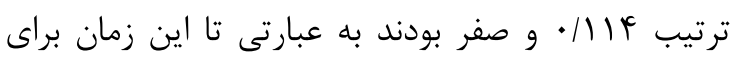
افرادى كه متاستاز را تجربه كرده بودند مرى رئ رخ ندادي احتمال كذار به متاستاز در يانزدهمين سال مطالعه

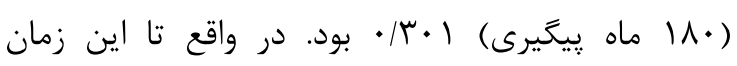

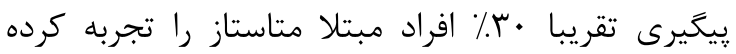
بودند. مقدار احتمال براى گذذار به حالت مركى در سال

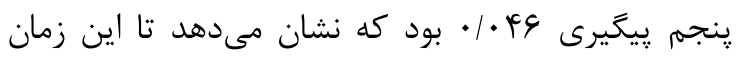

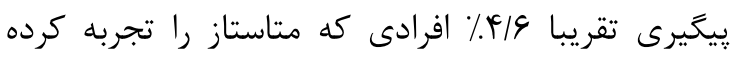
بودند فوت شدهاند. احتمال ماندن نيز نشان مىدهد كهان

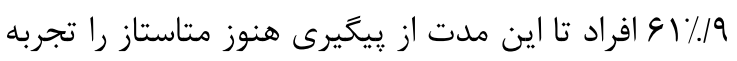
نكرده و زنده ماندهاند (جدول ؟). در نمودار 1 احتمال گذار بين هر رخداد بر اساس مانداندان يیيخيرى افراد از زمان تشخيص تا پايان مطالعه نشان داده

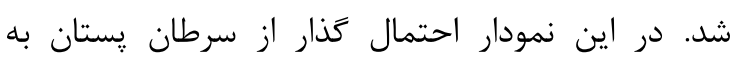
متاستاز در حال افزايش است در واقع در طول دورار إندان

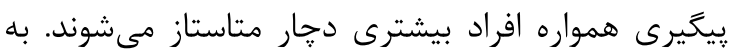

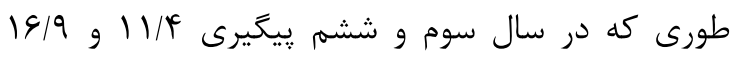

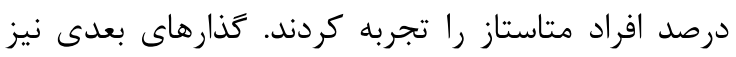
روند صعودى دارند اما احتمال كذار در آنها كمتر از

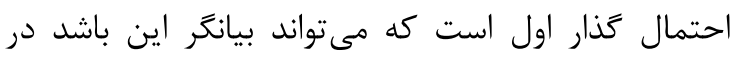

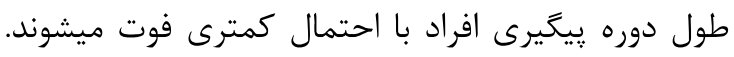
اين احتمال وجود دارد كه رخداد مركى براى افراد بيمار بعد از يايان يِيَيرى رخ داده است. نظر به اهميت سنجش

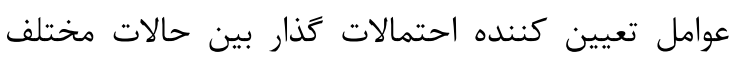
سرطان קستان در اين مطالعه از مدل ركرسيون مخاطره

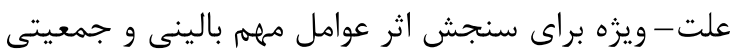
بر روند سير طبيعى سرطان يستان تا رخ داد مركى

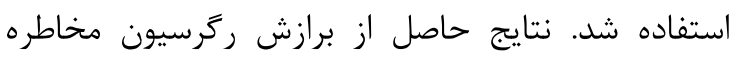

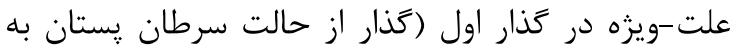

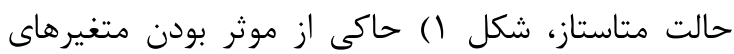

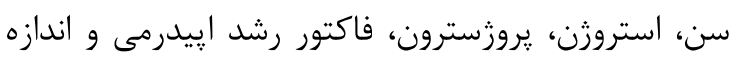
تومور در بيشرفت سرطان يستان به متاستاز بودند.
براى برآورد احتمال كذار بين حالتها در زمان t (تعداد

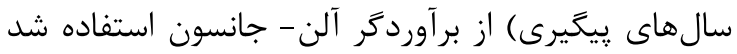

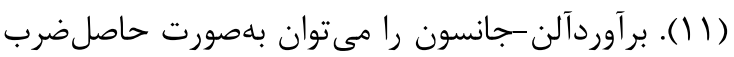
ماتريسى به شكل زير نوشت: $\widehat{\mathrm{P}}(\mathrm{s}, \mathrm{t})=\prod_{\mathrm{s}<\mathrm{u} \leq \mathrm{t}}\{\mathrm{I}+\widehat{\mathrm{d} \Lambda}(\mathrm{u})\}$ ماتريس همانى است و I

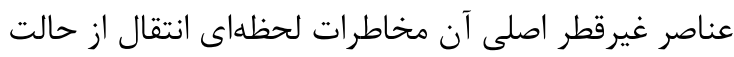

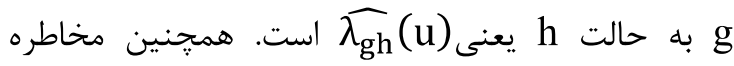
$\widehat{\lambda_{\mathrm{gh}}}(\mathrm{s})=\frac{\mathrm{d}_{\mathrm{gh}}(\mathrm{s})}{\mathrm{Y}_{\mathrm{g}}(\mathrm{s})}$

كه در آن Yg (S) تعداد افرادى است كه در زمان S در

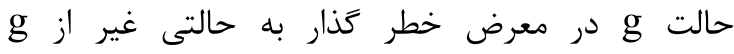

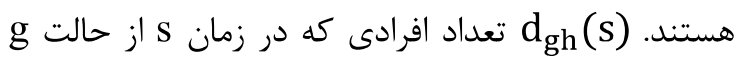

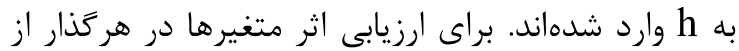

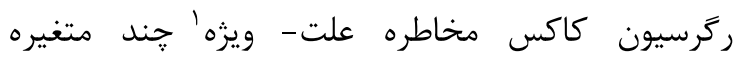

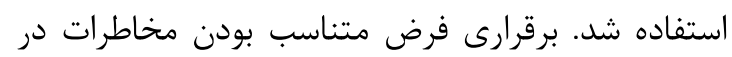

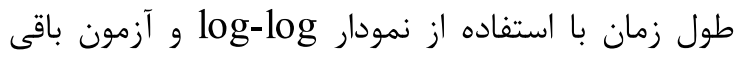
ماندهاى شونفلد تاييد خرديد. در ابتدا عوامل به صورت آندان تكى وارد مدل شدند و سيس اين عوامل با هم وارد مدل

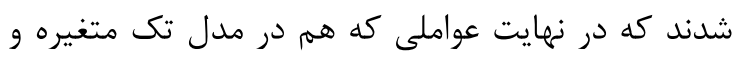

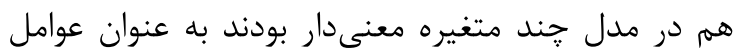

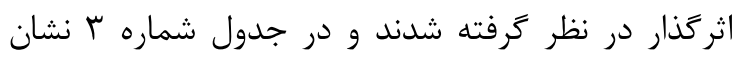

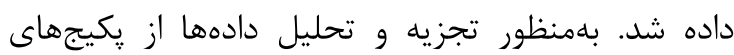

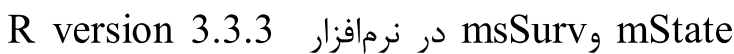

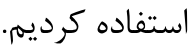

\section{يافتهها}

ميانگين سنى افراد شركت كننده در اين مطالعه در زمان

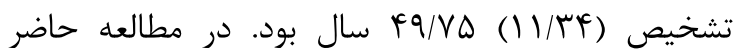

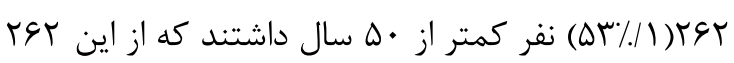

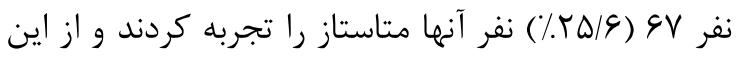

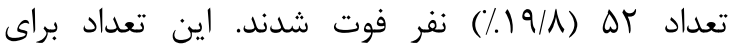

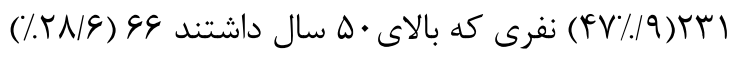

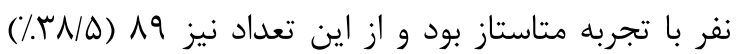

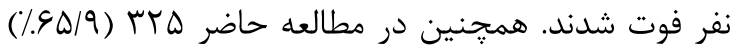
نفر سابقه انجام عمل جراحى برداشت پستان را داشتند كه

${ }^{1}$ Cox regression cause-specific hazard 
شكل ()، وضعيت استروزن و يروزسترون بودند. مخاطره

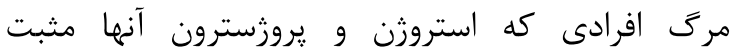
است نسبت به كسانى كه اين فاكتورها در آنها منفى

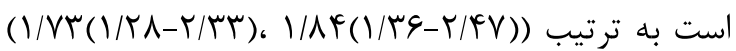
بود. ميزان بقاى افراد با تعداد كرههاى HR(\%95CI)= لنفاوى دركير رابطه مستقيمى دارد به طورى كه مخاطره

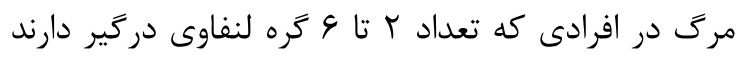

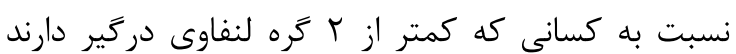
HR(\%95CI)=I/VQ(I/T-rr/Fq) كه مربوط به بيشرفت سرطان پستان به مرك است (شكل

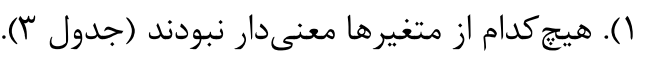

مخاطره وقوع متاستاز افرادى كه استروزن، يروزسترون

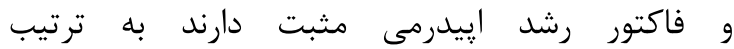

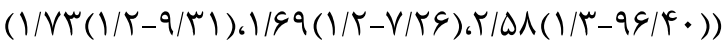
برابر افرادى كه اين فاكتورها در آنها HR(\%95CI)=

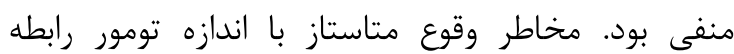
مستقيمى دارد به طورى كه اين مخاطره براى افرادى

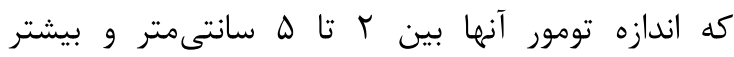
از ه هانتىمتر است نسبت به افرادى كه اندازه تومور

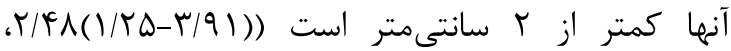
HR(\%95CI)=( (1/YA (1//-1•/V9) موثر بر حذار دوم (كذار از حالت متاستاز به حالت الت مرى

جدول ا: اطلاعات توصيفى متغيرهاى بالينى و زمينهاى

\begin{tabular}{|c|c|c|c|c|c|}
\hline $\begin{array}{c}\text { مرى } \\
(د ر ص د)\end{array}$ & متاستاز & $\begin{array}{c}\text { بدون متاستاز } \\
\text { (درصد) }\end{array}$ & تعداد(درصد) & كروه & متغير هاى بيشگَو \\
\hline$\Delta r(19 / \Lambda)$ & $G V(T \Delta / G)$ & $19 \Delta(V F / F)$ & TGT(DT/I) & كمتر از •ه سال & سن در زمان \\
\hline$\Lambda 9(\Gamma \wedge / \Delta)$ & $G 9(Y \wedge / 9)$ & $18 \Delta(V \mid / F)$ & TMI(FV/Q) & بيشتر و مساوى •D سال & تشخيص \\
\hline$r \mid(F \cdot / f)$ & $I T(Y \Delta)$ & $r q(\vee \Delta)$ & $\operatorname{ar}(r F / I)$ & برداشت تومور و جراحى با حفظ & \\
\hline $\operatorname{sf}(19 / \mathrm{V})$ & $\Lambda \Gamma(r \Delta / \Delta)$ & $r+q(V / / D)$ & $\operatorname{rr\Delta }(9 \Delta / 9)$ & برداشت پِتان & \\
\hline FI(Tr) & $\operatorname{Fr}(Y Y / \Lambda)$ & $I T K(V D / Y)$ & $\mid \vee \wedge(\Gamma \varepsilon / 1)$ & منفى & \multirow{2}{*}{ استروزن } \\
\hline$G T(Y G / V)$ & $\Delta V(Y Y / G)$ & $I V \Delta(V \Delta / Y)$ & TrT(FV/I) & مثبت & \\
\hline$\Delta V(T r / \Delta)$ & $\operatorname{Rr}(r g / \Delta)$ & $\operatorname{lr\Delta }(V / / \Delta)$ & $I V \cdot(T K / Q)$ & منفى & \multirow{2}{*}{ يروزسترون } \\
\hline$f \wedge(r \cdot / r)$ & $\Delta \Delta(Y T / T)$ & $\operatorname{lAr}(V \& \mid \Lambda)$ & $r T V(Y N / I)$ & مثبت & \\
\hline$f \mid(r \cdot / 1)$ & KQ(YT/I) & $1 \cdot 9(V \vee / 9)$ & $\mid r G(T V / G)$ & منفى & \multirow{2}{*}{ فاكتور رشد إيدرمى } \\
\hline$f \Delta(19 / \Delta)$ & $\Delta F(Y r / A)$ & $\operatorname{lV}(V \& / T)$ & (ז)(\$ & مثبت & \\
\hline$f \cdot(\mid \Lambda / q)$ & $\operatorname{Fr}(Y \cdot \mid \Lambda)$ & $19 \wedge(\vee 9 / r)$ & TIK(KT) & كمتر از ץ كره & \multirow{3}{*}{ تعداد كرههاى لنفاوى } \\
\hline$r V(r \Delta / r)$ & $f \Delta(r \mid / r)$ & $1 \cdot 1(9 N / V)$ & $\operatorname{lFV}(Y q / \Lambda)$ & بين r تا \& كره لنفاوى & \\
\hline $\operatorname{Fr}(q q / F)$ & $r \wedge(T \Delta / \Gamma)$ & $\Delta \Delta(\& \& / V)$ & $\Lambda \Delta(\mid V / T)$ & بيشتر از V Vره & \\
\hline$I r(r \Delta)$ & $\mid f(r \& / q)$ & $r \Lambda(V T / l)$ & $\Delta r(1 \cdot \mid \Delta)$ & كمتر از Y سانتىمتر & \multirow{3}{*}{ سايز تومور } \\
\hline$\Lambda r(Y G / \Gamma)$ & $V \wedge(\mid 9 / T)$ & YMI (VR/A) & rIr(\&\%/Q) & بين Y تا ه سانتىمتر & \\
\hline $\operatorname{IV}(19 / \mathrm{V})$ & $r Y(T G / F)$ & $G V(V / / Q)$ & $91(1 N / \Delta)$ & بيشتر از ه سانتىمتر & \\
\hline
\end{tabular}

جدول ז: احتمال كذار و ماندن در هر حالت بر اساس زمان بييگيرى

\begin{tabular}{|c|c|c|c|c|c|}
\hline \multirow[b]{2}{*}{ مرى } & \multicolumn{2}{|c|}{ احتمال ماندن } & \multicolumn{2}{|c|}{ احتمال كذار } & \multirow[b]{2}{*}{ زمان پيخيرى از زمان تشخيص(ماه) } \\
\hline & متاستاز & سرطان يستان & $r \rightarrow r$ & $\longrightarrow r$ & \\
\hline 1 & 1 & . /948 & . & $\cdot / \cdot \Delta r$ & $\pi$ \\
\hline 1 & 1 & $\cdot 19 \cdot r$ & . & $\cdot 1 \cdot \vee 9$ & rF \\
\hline 1 & 1 & - INGF & . &.$/ 11 f$ & re \\
\hline 1 & . /9v8 & $\cdot / \Lambda \cdot r$ & $\cdot / \cdot r F$ &.$/ 199$ & q. \\
\hline 1 & .1990 & $\cdot / V \cdot \Delta$ & ./.rs &.$/ K F \Delta$ & $\pi$. \\
\hline 1 &.$/ 9 \Delta F$ & .1919 & $\cdot 1 \cdot 48$ & $\cdot / \mu \cdot 1$ & 11. \\
\hline
\end{tabular}




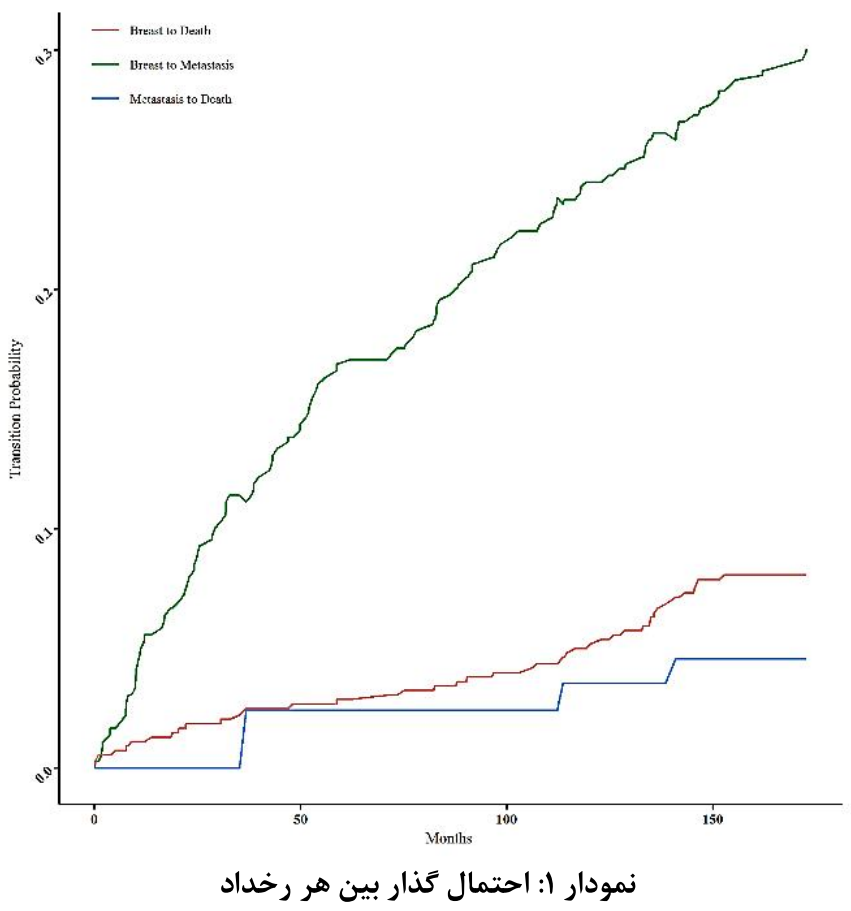

جدول با: نتايج مدل كاكس براى هر يك از متغيرهاى ييشَّو دموكرافيك و بالينى بر اساس كذار

\begin{tabular}{|c|c|c|c|c|c|c|c|}
\hline \multicolumn{2}{|c|}{ 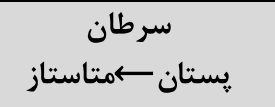 } & \multicolumn{2}{|c|}{ متاستاز ๘مرى } & \multicolumn{2}{|c|}{ 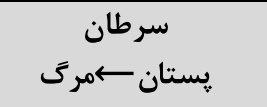 } & \multirow{2}{*}{ كروه } & \multirow{2}{*}{ متغير بِيشكَو } \\
\hline CI \%95 & HR & CI \%95 & HR & CI $\% 95$ & HR & & \\
\hline- & 1 & - & 1 & - & 1 & كمتر از • • سال & سن در زمان \\
\hline •/I-rT/AN & $1 / \Delta r$ & $\cdot / 1-\wedge \& / \curlyvee \wedge$ & $1 / \cdot \Delta$ & $\cdot / r-Y F / T$ & $1 / \Lambda$ & بيشتر و مساوى •D سال & تشخيص \\
\hline- & 1 & - & 1 & - & 1 & برداشت تومور و جراحى با حفظ & \\
\hline$\cdot / r-1 \Delta / \Delta r$ & $1 / 1 F$ & $\begin{array}{l}f-/ 91 \\
1 / 44\end{array}$ & $r / F G$ & - $/ \mathcal{E}-r T / \Delta \varphi$ & $r / T$ & برداشت يستان & إحنى \\
\hline- & 1 & - & 1 & - & 1 & منفى & \\
\hline$|/ r-r q / \Gamma|$ & INR & $\begin{array}{l}1 / 4 \lambda \\
r-/ 4 r\end{array}$ & $1 / V r$ & $\cdot / \Gamma-\Delta V / V{ }^{\epsilon}$ & $1 / r$ & مثبت & استروزن \\
\hline $\begin{array}{c}- \\
1 / T-V / T G\end{array}$ & $\begin{array}{l}1 \\
1 / 99\end{array}$ & $\begin{array}{c}- \\
1 / r-r q / F V\end{array}$ & $\begin{array}{l}1 \\
1 / \lambda F\end{array}$ & $\frac{-}{\cdot r-f \cdot / f}$ & $\begin{array}{l}1 \\
1 / 9\end{array}$ & مثنى مثبت & يروزسترون \\
\hline- & 1 & - & 1 & - & 1 & منفى & \\
\hline $1 / r-94 / 4$. & $r / \Delta \Lambda$ & $\begin{array}{l}r-/ V T \\
T / I F\end{array}$ & r/Ar & 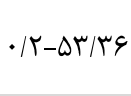 & $r / T \Delta$ & مثبت & فاكتور رشد إيدرمى \\
\hline- & 1 & - & 1 & - & 1 & كمتر از r كره & \\
\hline $1 / 1-\cdot r / \Delta r$ & $1 / 19$ & $\begin{array}{l}1 / 4 r \\
r-/ 4 q\end{array}$ & $1 / V \Delta$ & $\cdot / r-T V / G$ & $1 / r$ & بين r تا و كره لنفاوى & تعداد كرههاى لنفاوى \\
\hline $1 / r-r r / \cdot q$ & T/VD & $\begin{array}{l}1-/ \Delta r \\
. / 1 V\end{array}$ & $1 / \cdot 9$ & $\cdot / r-r N / 9$ & $1 / 4$ & بيشتر از ل كره & \\
\hline- & 1 & - & 1 & - & 1 & كمتر از Y سانتىمتر & \\
\hline $1 / 1-1 \cdot / 199$ & $1 / \Gamma \wedge$ & $\begin{array}{c}1-1 \cdot r \\
\cdot / 1-r \& / r \Lambda\end{array}$ & $\cdot / \Delta V$ & $\cdot / r-\mid \Delta / 1$ & $r / F$ & بين r تا ه سانتى متر & سايز تومور \\
\hline $1 / r-r \Delta / q 1$ & $r / F \Lambda$ & $\begin{array}{l}\cdot \mu \cdot \pi \\
i-1 \cdot T\end{array}$ & $\cdot 1 \Delta \Delta$ & $\cdot / 4-9 \cdot / 1$ & $r / T$ & بيشتر از ه سانتىمتر & \\
\hline
\end{tabular}


مىشود. لذا اين عامل مى تواند احتمال بزرى بودن تومور

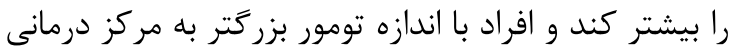
مراجعه كنند كه احتمال بروز متاستاز در اين افراد بيشتر

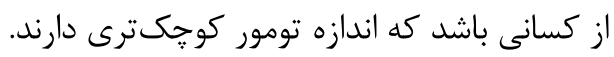

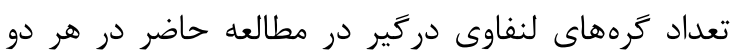

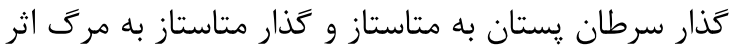

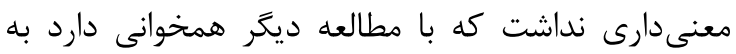

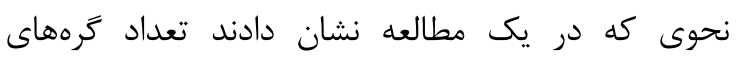

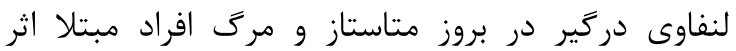

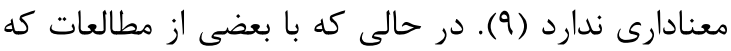

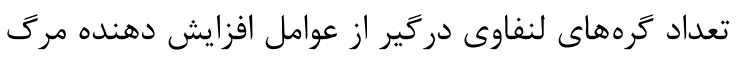

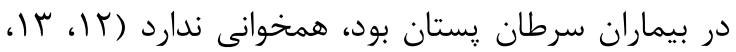
11). دليل اين تناقض مىتواند به دليل وضعيت بيماران

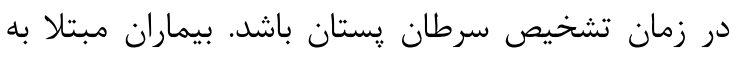

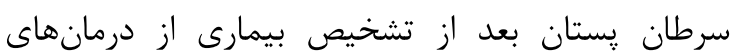
رزيمى و جراحى استفاده مى كنند. در مطالعه حاضر اثر

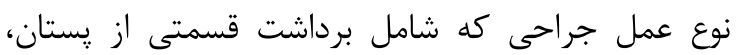

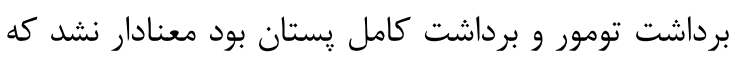

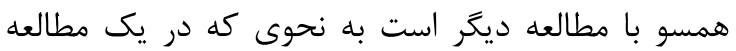

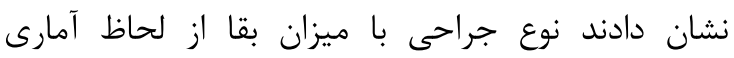

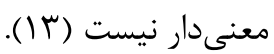
در مطالعه حاضر كيرندههاى هورمونى استروزن، يروزسترون و فاكتور رشد إيدرمى در وقوع متاستاز موثر

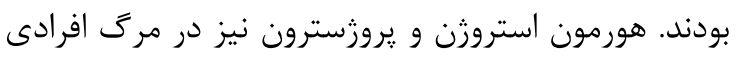

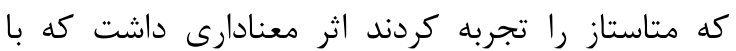
مطالعات ديخر همخوانى دارد به كونهاى كه در خُندين

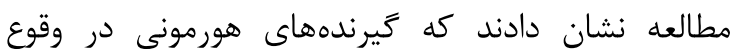

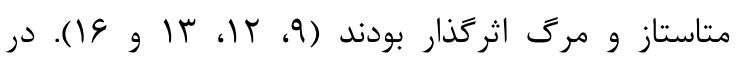
مطالعه ما افرادى كه وضعيت استروزن و فاكتور رشد

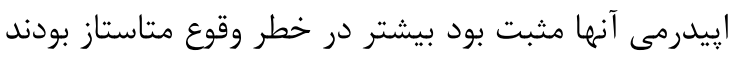

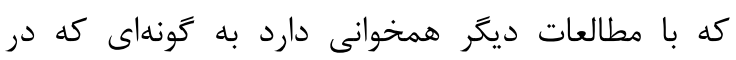
جندين مطالعه نشان دادند كه خطر مركى در بيمارانى كه

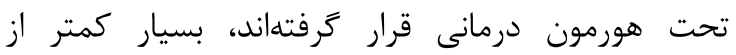

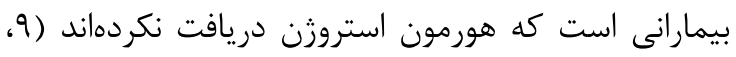

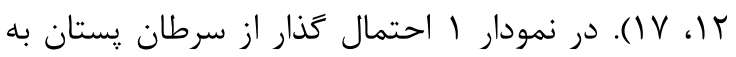

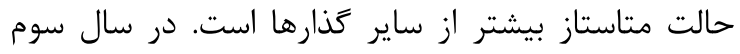

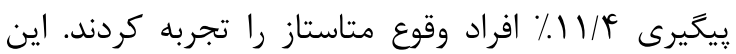

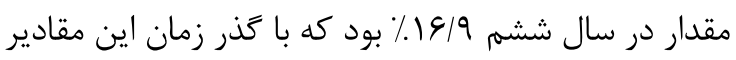

نتايج اين مطالعه نشان داد كه مدلهاى جند حالتى

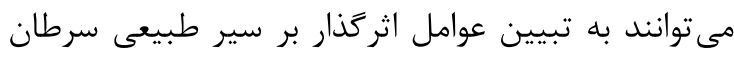

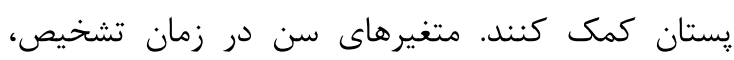

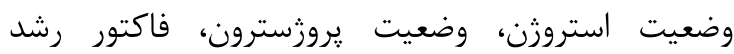

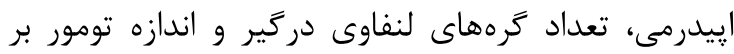
وقوع متاستاز موثر بودند. همجنين در سال سوم و ششمه

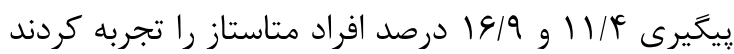
كه در طول دوره يِيخيرى همواره افراد بيشترى دجار

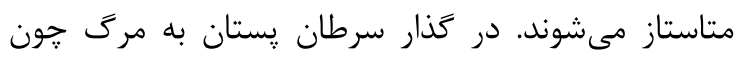

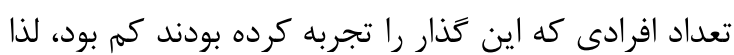

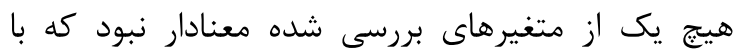

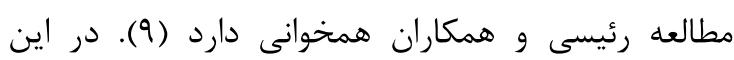

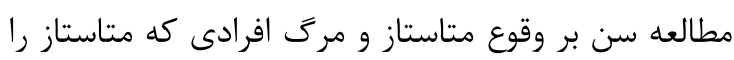
تجربه كرده بودند موثر نبود كه با مطالعات ديخر همخوانى مبر مباسي

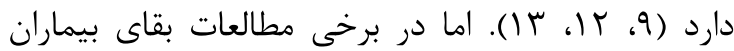

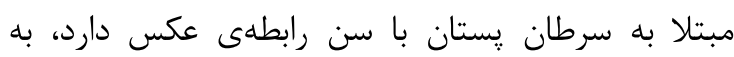

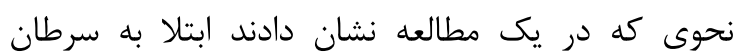
يستان در سنين بالا باعث كاهش بقاى بيمارى مىشود

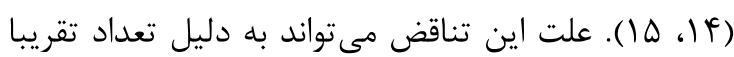

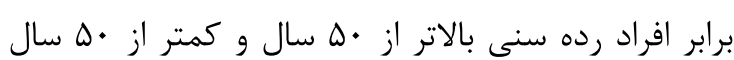
در مطالعه حاضر مربوط باشد.

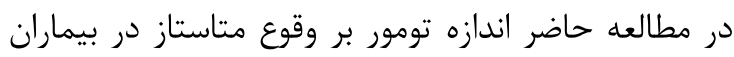

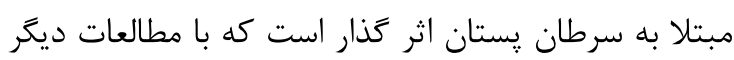

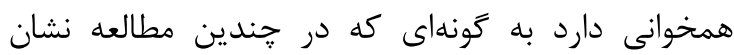

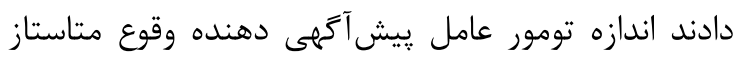

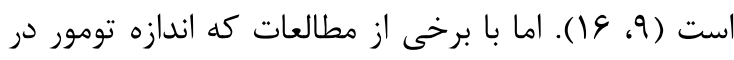

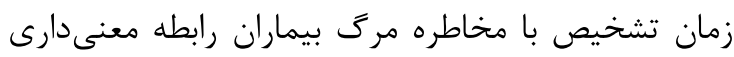

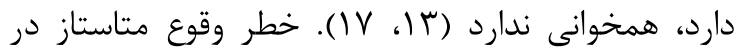

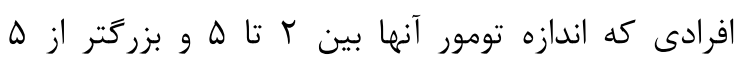

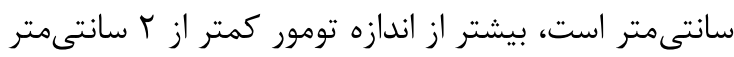
است. اين مقدار مخاطره وقوع متاستاز در مطالعات ديكر

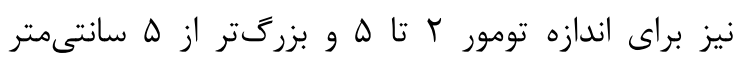

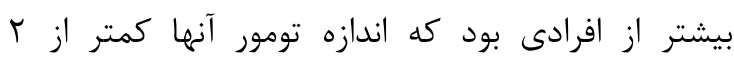
سانتىمتر بود (9، • (). از انجا كه افراد مبتلا به سرطان

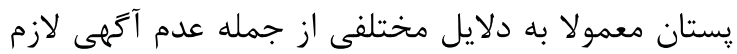
نسبت به بيمارى، ترس از بيمارى، عدم وجود امكانات لازم

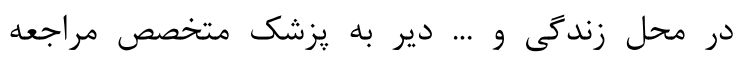

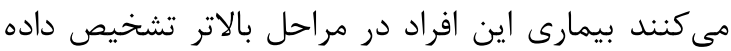




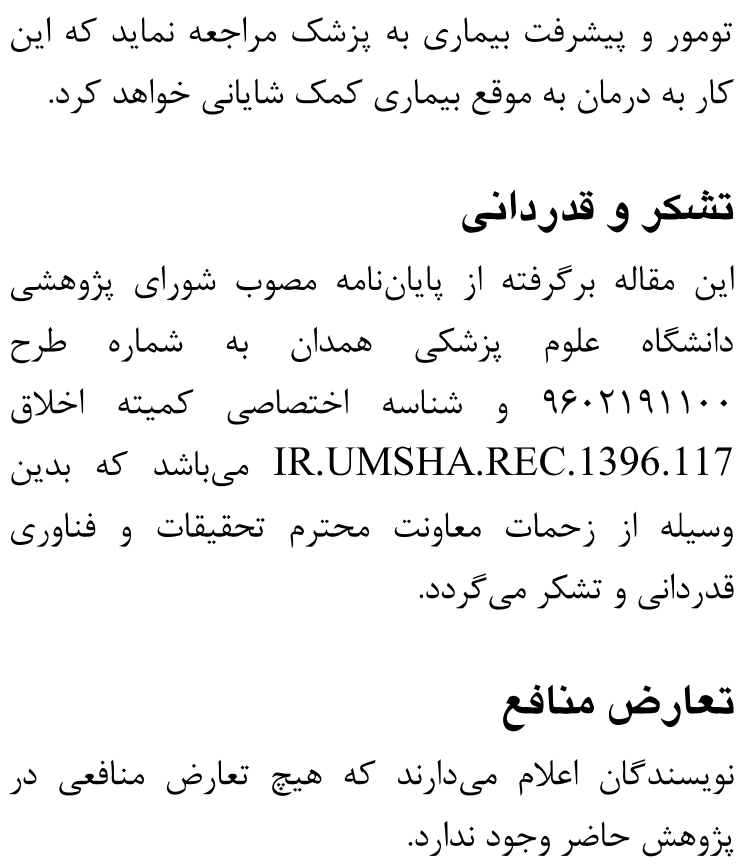

\section{References}

1. Kazemi A, Eskandari O, Amin MM, Nesaee P. A Survey on breast cancer status in kurdistan province on medical geography viewpoint during 2006 - 2010. health system research. 2015; 11(3).

2. Mahboobi A, Alvandi S, Alizadeh Navaei R. An analytical survey on breast lesions in mammography. Journal of Babol University Of Medical Sciences. 2004;6(2):52-5.

3. Mahmood Reza G, Zeinab Moghadami F, Jamileh A, Mehdi M, Parisa M. Prognostic factors of metastases in breast cancer patients using the recurrent Andersen-Gill model. Koomesh. 2013;14(4):483-9.

4. Klein JP, Moeschberger ML. Survival analysis: techniques for censored and truncated data. Springer Science \& Business Media. 2006; 8(5):80-115.

5. Hosmer DW LS. Applied Survival Analysis: Regression Modelling of Time to Event Data . Eur Orthodontic Soc. 1999;7(2):561-2.

6. Vahdaninia M, Harirchi A.M, Montazeri A. Fiveyear survival in Iranian breast cancer patients: a prospective study. Health Monitor Journal of the Iranian Institute for Health Sciences Research. 2003; 2(2):147-54.

7. Najafi R, Amiri F, Roshanaei G, Abbasi M, Razi M. Factors Affecting Survival in Patients with Breast Cancer in Hamedan Province from 2001

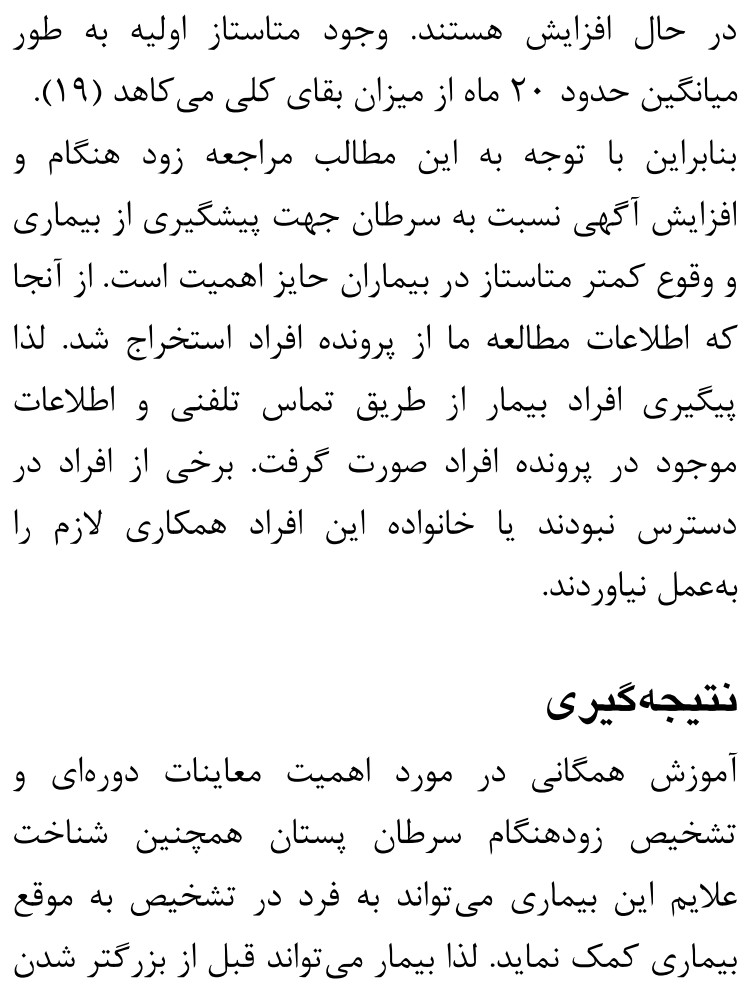

to 2018. Iranian Quarterly Journal of Breast Diseases. 2019;12(4):31-8.

8. mohamadali Noruzi M, Hosseni AF, Gohari MR . Application of multilevel model in determining the effective factors in the length of stay among appendectomy patients. Razi Journal of Medical Sciences. 2014;20(115):70-7.

9. Raesizadeh M, Seghatoleslami M, Hoseinzade M, Saki Malehi A. Survival Analysis of Breast Cancer Patients According to Intermediate and Endpoint Events: Applying Illness-Death Model. Iranian Journal of Epidemiology. 2018; 13(4):291-8.

10. Putter H, van der Hage J, de Bock GH, Elgalta $\mathrm{R}$, van de Velde CJ. Estimation and prediction in a multi-state model for breast cancer. Biometrical journal Biometrische Zeitschrift. 2006; 48(3):366-80.

11. Kim J. Ronald B. Geskus, Data Analysis with Competing Risks and Intermediate States. New York: Chapman and Hall/CRC. Biometrics. 2015; 71(4):1197-8.

12. Moghadami Fard Z, abolghasemi J, asgaridarian A, Gohari MR. Survival analysis of patients with breast cancer using the Aalen's additive hazard model. Journal of North Khorasan University of Medical Sciences. 2012; 3(5):171-9. 
13. Faradmal J, Mafi M, Sadighi-Pashaki A, Karami M, Roshanaei G. Factors Affecting Survival in Breast Cancer Patients Referred to the Darol Aitam-e Mahdieh Center. Journal of Advances in Medical and Biomedical Research. 2014; 22(93):105-15.

14. Lakzaei M, Salarilak S, Khalkhali HR ,Maleki $\mathrm{D}$, Esnaashari O. Association between age of morbidity and prognosis of breast cancer. Studies in Medical Sciences. 2015;26(7):62533.

15. Kasaeian A, Abadi A, Mehrabi Y, Mousavi Jarrahi A. Estimating Relative Survival of Breast Cancer Patients Referring to Imam Khomeini Cancer Institute during 1990-95. 2009; 20(6):1-7.

16. Gohari MR, Moghadami fard Z, Abolghasemi J, Mohammadi M, Mokhtari p. Prognostic factors of metastases in breast cancer patients using the recurrent Andersen-Gill model. koomesh. 2019; 14(4)483-9.

17. Atashgar $\mathrm{K}$, Molana SH, Biglarian A, Sheikhaliyan A. Analysis of Survival Data of Patient with Breast Cancer Using Cox Regression Model. Iranian Journal of Surgery. 2016; 24(1):62.

18. Haghighat S. Survival rate and its correlated factors in breast cancer patients referred to Breast Cancer Research Center. Iranian Quarterly Journal of Breast Diseases. 2013; 6(3):28-36.

19. Fariborz M, Shirin M, Arash R. Relations of Disease-Free Survival and Overall Survival with Age and Primary Metastases in Patients with Breast Cancer. Journal Of Isfahan Medical School. 2013; 31(225):1. 